Korean J. Math. 20 (2012), No. 2, pp. 161-176

\title{
NEW CONSTRUCTION OF THE EAGON-NORTHCOTT COMPLEX
}

\author{
OH-Jin Kang AND JoOHyUng KIM*
}

\begin{abstract}
The authors [6] introduced the concept of a complete matrix of grade $g>3$ to describe a structure theorem for complete intersections of grade $g>3$. We show that a complete matrix can be used to construct the Eagon-Northcott complex [7]. Moreover, we prove that it is the minimal free resolution $\mathbb{F}$ of a class of determinantal ideals of $n \times(n+2)$ matrices $X=\left(x_{i j}\right)$ such that entries of each row of $X=\left(x_{i j}\right)$ form a regular sequence and the second differential map of $\mathbb{F}$ is a matrix $f$ defined by the complete matrices of grade $n+2$.
\end{abstract}

\section{Introduction}

Let $k$ be a field containing the field $\mathbb{Q}$ of rational numbers and let $R=k\left[x_{i j} \mid 1 \leq i \leq m, 1 \leq j \leq n\right]$ be the polynomial ring over a field $k$ with indeterminates $x_{i j}$. Eagon and Northcott [7] defined a free complex from a matrix over a commutative ring with identity which is a generalization of the standard Koszul complex. As an application of it, they constructed the minimal free resolution of $R / I_{t}(X)$, where $t=\min (m, n)$. Also Buchsbaum and Rim [4] separately constructed the minimal free resolution of the class of the determinantal ideals. Buchsbaum [5] used the multilinear algebra to give other version of the Eagon and Northcott complex. Buchsbaum and Eisenbud [2] noted that the Eagon-Northcott and Buchsbaum-Rim complexes are constructed by the multilinear algebra, that is, the complexes are described in terms of tensor products of

Received March 12, 2012. Revised June 1, 2012. Accepted June 5, 2012.

2010 Mathematics Subject Classification: 13C40, 13D02.

Key words and phrases: complete matrix of grade $g$, minimal free resolution, determinantal ideal.

This paper was supported by Wonkwang University in 2011.

*Corresponding author. 
exterior, symmetric and divided power algebras. On the other hand, using the representation theory of the general linear groups, Lascoux [12], Pragacz and Weyman [13], and Roberts [14] constructed the minimal free resolution of $R / I_{t}(X)$ for any $m, n, t$, where $R$ contains the field $\mathbb{Q}$ of the rational numbers. Akin, Buchsbaum and Weyman [1] developed the characteristic free representation theory of the general linear groups and constructed the minimal free resolution of $R / I_{t}(X)$ over $R=\mathbb{Z}$ in the case of $t=\min (m, n)-1$. Roberts [14] proved that there exists a minimal free resolution of $R / I_{t}(X)$ over $R=\mathbb{Z}$ if and only if the Betti numbers of $R / I_{t}(X)$ is independent of the characteristic of the base field. Hashimoto and Kurano [10] used this proof to show that there exists a minimal free resolution of $R / I_{t}(X)$ over $R=\mathbb{Z}$ in the case of $m=n=t+2$. Hishimoto $[8,9]$ also extended this result to the case of $t=\min (m, n)-2$ and proved that there is no minimal free resolution of $R / I_{t}(X)$ over $R=\mathbb{Z}$ in the case of $2 \leq t \leq \min (m, n)-3$. Recently, Kang and Ko [11] introduced a complete matrix of grade 4 to describe a structure theorem for the complete intersections of grade 4 and Choi, Kang and Ko [6] extended this to a structure theorem for the complete intersections of grade $g>3$. In this paper, we introduce a matrix $f$ defined by complete matrices $f(i)$ of grade $n+2$,

$$
f=\left[\begin{array}{llllll}
f(1)^{t} & -f(2)^{t} & \cdots & (-1)^{i+1} f(i) & \cdots & (-1)^{n+1} f(n)^{t}
\end{array}\right]
$$

and define the ideal $\mathcal{D}_{n+1}(f)$ associated with $f$, which is generated by the maximal minors of the $n \times(n+2)$ matrix $D(f)=\left(x_{i j}\right)$, where $x_{i j}$ is the $(n+1)$ st root of the $j$ th $(n+1) \times(n+1)$ diagonal submatrix $S_{i j}$ of the complete matrix $f(i)$ of grade $n+2$.

The main purpose of this paper is to construct a minimal free resolution $\mathbb{F}$ of a class of the determinantal ideals generated by the maximal minors of an $n \times(n+2)$ matrix $D(f)$, such that entries of each row of $D(f)$ form a regular sequence and the second differential map of $\mathbb{F}$ is a matrix $f$ defined by the complete matrices of grade $n+2$. This method gives us a case of constructing the minimal free resolution of a class of the determinantal ideals of an $n \times(n+2)$ matrix. Among classes of determinantal ideals generated by the maximal minors of $p \times q$ matrices $Y=\left(y_{i j}\right)$ with $p<q$ and indeterminates $y_{i j}$, except the class mentioned above it is not easy to find one of them which has the minimal free resolution such that the second differential map of it has a matrix defined by complete matrices of grade $q$ and each row of $Y$ forms a regular sequence of length $q$. 


\section{A minimal free resolution of a class of determinantal ideals}

Let $k$ be a field containing the field $\mathbb{Q}$ of rational numbers and let $R=k\left[x_{i j} \mid 1 \leq i \leq n, 1 \leq j \leq n+2\right]$ be the polynomial ring over a field $k$ with indeterminates $x_{i j}$. Choi, Kang and Ko [6] introduced a complete matrix of grade $g$ to describe a structure theorem for complete intersections of grade $g>3$. Choi, Kang and Ko [6] also showed that the second differential map of the Koszul complex defined by a regular sequence $\mathbf{x}=x_{1}, x_{2}, \ldots, x_{g}$ satisfies the conditions of Proposition 4.3 and Theorem 4.4 [6]. By using them and the induction on $g>3$ we can define a complete matrix of grade $g>3$ from the second differential map of the Koszul complex defined by a regular sequence $\mathbf{x}$. Theorem 4.4 [6] enables us to define a complete matrix of grade $g$. By the induction on $g$, we call $\bar{T}_{k}$ given in Theorem 4.4 [6] a complete matrix of grade $g-1$ for each $k$. For more background information we refer the reader to $[6,11]$.

DeFinition 2.1. [6] Let $R$ be a commutative ring with identity. Let $g>3$ and $t=\left(\begin{array}{l}g \\ 2\end{array}\right)$ be integers. A $g \times t$ matrix $f$ over $R$ is said to be complete of grade $g$ if

(1) $f$ has $g$ disjoint pairs $(S, T)$ of a $g \times(g-1)$ submatrix $S$ and a $g \times(t-g+1)$ submatrix $T$;

(2) By removing a row and interchanging columns, each pair $(S, T)$ can be reduced to a pair $(\bar{S}, \bar{T})$, where $\bar{S}$ is a $(g-1) \times(g-1)$ diagonal matrix with $\operatorname{det}(\bar{S})=x^{g-1}$ for some $x$ in $R$, up to sign, and $\bar{T}$ is the complete matrix of grade $g-1$ with grade $\mathcal{K}_{g-2}(\bar{T})=g-1$.

Let $n$ be an integer with $n \geq 2$ and $x_{i 1}, x_{i 2}, \ldots, x_{i n+2}$ a regular sequence on $R$ for $i=1,2, \ldots, n$. First we construct a complete matrix of grade $n+2$. Let $j$ and $k$ be integers with $1 \leq j \leq n+1$ and $1 \leq k \leq n+2$, respectively. We define $f(i, j, k)$ to be a $1 \times(n+2-j)$ matrix whose the $l$ th entry is given by

$$
f(i, j, k)_{l}= \begin{cases}(-1)^{j+1} x_{i j} & \text { if } j<k \text { and } l=k-j \\ 0 & \text { if } j<k \text { and } l \neq k-j \\ (-1)^{l+j} x_{i j+l} & \text { if } j=k \\ 0 & \text { if } j>k .\end{cases}
$$

Then we observe easily from (2.1) that if $j>k$, then $f(i, j, k)$ is a zero matrix and, if $j=k$, then $f(i, j, k)$ has the form of

$$
f(i, j, k)=\left[\begin{array}{llll}
(-1)^{1+j} x_{i 1+j} & (-1)^{2+j} x_{i 2+j} & \cdots & (-1)^{n+2} x_{i n+2}
\end{array}\right],
$$


and, if $j<k$, then the $(k-j)$ th entry of $f(i, j, k)$ is $(-1)^{j+1} x_{i j}$ and other entries are equal to zero. Let $s=\left(\begin{array}{c}n+2 \\ 2\end{array}\right)$. Define $f(i, k)$ to be an $s \times 1$ matrix given by

$$
f(i, k)=\left[\begin{array}{llll}
f(i, 1, k) & f(i, 2, k) & \cdots & f(i, n+1, k)
\end{array}\right]^{t} .
$$

We also define $f(i)$ to be an $(n+2) \times s$ matrix given by

$$
f(i)=\left[\begin{array}{llll}
f(i, 1) & f(i, 2) & \cdots & f(i, n+2)
\end{array}\right]^{t} .
$$

The following theorem shows that $f(i)$ is a complete matrix of grade $n+2$.

TheOREM 2.2. With notations as above, we have

(1) Every row of $f(i)$ has exactly $(n+1)$ nonzero entries.

(2) Every column of $f(i)$ has exactly two nonzero entries.

(3) Pairs of positive integers which represent the positions of the two nonzero entries in any two columns of $f(i)$ are all distinct.

Proof. (1) It suffices to show that $f(i, k)$ has exactly $(n+1)$ nonzero entries for each $k$. It follows from (2.1) that if $k=1$ and $j=k$, then every entry of $f(i, j, k)$ is nonzero and if $j>k$, then $f(i, j, k)$ is a zero matrix. Hence we can get from (2.2) that the number of nonzero entries of $f(i, 1)^{t}$ is equal to $n+1$. It follows from (2.1) that if $k>1$, then the number of nonzero entries of $f(i, j, k)$ is equal to 1 for $j<k$ and every entry of $f(i, k, k)$ is nonzero. Moreover $f(i, j, k)$ is a zero matrix for $j>k$. Hence the number of nonzero entries of $f(i, k)$ is equal to $n+1$.

(2) It follows from (2.2) and (2.3) that if $\mathbf{r}_{k}(f(i))$ is the $k$ th row of $f(i)$, then we have

$$
\mathbf{r}_{k}(f(i))=\left[\begin{array}{llll}
f(i, 1, k) & f(i, 2, k) & \cdots & f(i, n+1, k)
\end{array}\right] .
$$

Let $\mathbf{c}_{l}(f(i))$ be the $l$ th column of $f(i)$. We show that the number of nonzero entries of $\mathbf{c}_{l}(f(i))$ is equal to 2. We observe from (2.1) that if $l$ is an integer with $1 \leq l \leq n+1$, then the first and $(l+1)$ th entries of $\mathbf{c}_{l}(f(i))$ are nonzero and other entries are zero: if $l$ is an integer with $n+2 \leq l \leq 2 n+1$, then the second and $(l-(n+1)+2)$ th entries of $\mathbf{c}_{l}(f(i))$ are nonzero and other entries are zero. Continuing this way, we get the following : if

$$
\phi(m)=\sum_{q=1}^{m-1}(n+2-q)
$$


and if $l$ is an integer with $1+\phi(h) \leq l \leq \phi(h+1)$ for $h=1,2, \ldots, n+1$, then the $h$ th and $(l-\phi(h)+h)$ th entries of $\mathbf{c}_{l}(f(i))$ are nonzero and other entries are zero. Thus we get the desired result.

(3) It follows from the observation in part (2).

We describe a submatrix of an $m \times n$ matrix $h$ for the following example. Let $h\left(i_{1}, i_{2}, \ldots, i_{p} \mid j_{1}, j_{2}, \ldots, j_{q}\right)$ be the $p \times q$ submatrix of $h$ consisting of the $p q$ entries at the intersection of rows $i_{1}, i_{2}, \ldots, i_{p}$ with columns $j_{1}, j_{2}, \ldots, j_{q}$, where $1 \leq i_{1}<i_{2}<\cdots<i_{p} \leq m$ and $1 \leq j_{1}<j_{2}<\cdots<j_{q} \leq n$.

Now we give an example to illustrate Theorem 2.2.

EXAMPLE 2.3. Let $x_{i 1}, x_{i 2}, \ldots, x_{i 4}$ be a regular sequence on a commutative ring with identity. For $i=1,2$, we define $f(i, j, k)$ to be a $1 \times(4-j)$ matrix as follows

$$
\begin{aligned}
& f(i, 1,1)=\left[\begin{array}{lll}
x_{i 2} & -x_{i 3} & x_{i 4}
\end{array}\right], \quad f(i, 2,1)=\left[\begin{array}{ll}
0 & 0
\end{array}\right], \quad f(i, 3,1)=[0], \\
& f(i, 1,2)=\left[\begin{array}{lll}
x_{i 1} & 0 & 0
\end{array}\right], \quad f(i, 2,2)=\left[\begin{array}{ll}
-x_{i 3} & x_{i 4}
\end{array}\right], \quad f(i, 3,2)=[0], \\
& f(i, 1,3)=\left[\begin{array}{lll}
0 & x_{i 1} & 0
\end{array}\right], \quad f(i, 2,3)=\left[\begin{array}{ll}
-x_{i 2} & 0
\end{array}\right], f(i, 3,3)=\left[x_{i 4}\right], \\
& f(i, 1,4)=\left[\begin{array}{lll}
0 & 0 & x_{i 1}
\end{array}\right], \quad f(i, 2,4)=\left[\begin{array}{ll}
0 & -x_{i 2}
\end{array}\right], \quad f(i, 3,4)=\left[x_{i 3}\right] .
\end{aligned}
$$

Then we have

$$
\begin{aligned}
f(i, 1) & =\left[\begin{array}{llllll}
x_{i 2} & -x_{i 3} & x_{i 4} & 0 & 0 & 0
\end{array}\right]^{t}, \quad f(i, 2)=\left[\begin{array}{llllllll}
x_{i 1} & 0 & 0 & -x_{i 3} & x_{i 4} & 0
\end{array}\right]^{t}, \\
f(i, 3) & =\left[\begin{array}{lllllll}
0 & x_{i 1} & 0 & -x_{i 2} & 0 & x_{i 4}
\end{array}\right]^{t}, \quad f(i, 4)=\left[\begin{array}{llllll}
0 & 0 & x_{i 1} & 0 & -x_{i 2} & x_{i 3}
\end{array}\right]^{t}
\end{aligned}
$$

and

$$
f(i)=\left[\begin{array}{l}
f(i, 1)^{t} \\
f(i, 2)^{t} \\
f(i, 3)^{t} \\
f(i, 4)^{t}
\end{array}\right]=\left[\begin{array}{cccccc}
x_{i 2} & -x_{i 3} & x_{i 4} & 0 & 0 & 0 \\
x_{i 1} & 0 & 0 & -x_{i 3} & x_{i 4} & 0 \\
0 & x_{i 1} & 0 & -x_{i 2} & 0 & x_{i 4} \\
0 & 0 & x_{i 1} & 0 & -x_{i 2} & x_{i 3}
\end{array}\right]
$$

It is easy to show that $f(i)$ is a complete matrix of grade 4 for each $i$. We observe that every row of $f(i)$ contains exactly three nonzero and three zero entries. For $k=1,2,3,4$, let $S_{k}$ be the $4 \times 3$ submatrix of $f(i)$ formed by the three columns which entries of the $k$ th row of $f(i)$ are nonzero and let $T_{k}$ be the $4 \times 3$ submatrix of $f(i)$ formed by the three 
columns which entries of the $k$ th row of $f(i)$ are zero. That is,

$$
\begin{array}{ll}
S_{1}=f(i)(1,2,3,4 \mid 1,2,3), & T_{1}=f(i)(1,2,3,4 \mid 4,5,6), \\
S_{2}=f(i)(1,2,3,4 \mid 1,4,5), & T_{2}=f(i)(1,2,3,4 \mid 2,3,6), \\
S_{3}=f(i)(1,2,3,4 \mid 2,4,6), & T_{3}=f(i)(1,2,3,4 \mid 1,3,5), \\
S_{4}=f(i)(1,2,3,4 \mid 3,5,6), & T_{4}=f(i)(1,2,3,4 \mid 1,2,4) .
\end{array}
$$

Let $\bar{S}_{k}$ be the $3 \times 3$ submatrix of $S_{k}$ obtained by deleting the $k$ th row of $S_{k}$ for each $k$. Then $\bar{S}_{k}$ is a diagonal matrix with determinant $x_{k}^{3}$. Let $\bar{T}_{k}$ be the $3 \times 3$ matrix obtained by exchanging the first and third columns of $T_{k}$ and by deleting the $k$ th row of $T_{k}$. Then $\bar{T}_{k}$ becomes an alternating matrix when the second column is multiplied by -1 , and $\operatorname{Pf}_{2}\left(\mathcal{A}\left(\bar{T}_{k}\right)\right)$ has grade 3 for each $k$. ( The definition of $\mathcal{A}\left(\bar{T}_{k}\right)$ has appeared in (3.1) [11].) Hence $f(i)$ is a complete matrix of grade 4 . Actually it is the second differential map in the Koszul complex defined by a regular sequence $\mathbf{x}_{i}=x_{i 1},-x_{i 2}, x_{i 3},-x_{i 4}$.

The following proposition plays an important role in defining an $\left(\begin{array}{c}n+2 \\ 2\end{array}\right) \times$ $n(n+2)$ matrix defined by complete matrices of grade $n+2$.

Proposition 2.4. With notations as above, if $x_{i 1}, x_{i 2}, \ldots, x_{i n+2}$ is a regular sequence on $R$ for each $i$, then $f(i)$ is a complete matrix of grade $n+2$.

Proof. It suffices to show that the conditions of Proposition 4.3 and Theorem 4.4 [6] are satisfied. Since $x_{i 1}, x_{i 2}, \ldots, x_{i n+2}$ is a regular sequence for each $i$, so is $x_{i 1},-x_{i 2}, x_{i 3},-x_{i 4}, \ldots,(-1)^{k+1} x_{i k}, \ldots,(-1)^{n+3} x_{i n+2}$. Thus

$$
\mathbf{y}_{i 1}=-x_{i 2}, x_{i 3},-x_{i 4}, \ldots,(-1)^{k+1} x_{i k}, \ldots,(-1)^{n+3} x_{i n+2}
$$

is a regular sequence. We observe that $f(i)$ has the form

$$
\begin{aligned}
& f(i)=\left[\begin{array}{cc}
l_{1}(i) & \mathbf{0} \\
\hline l_{2}(i) & f(i)_{1}
\end{array}\right], \\
& l_{1}(i)=\left[\begin{array}{lllllll}
-x_{i 2} & x_{i 3} & -x_{i 4} & \cdots & (-1)^{k+1} x_{i k} & \cdots & (-1)^{n+3} x_{i n+2}
\end{array}\right], \\
& l_{2}(i)=\operatorname{diag}\left\{x_{i 1}, x_{i 1}, \cdots, x_{i 1}\right\} \\
& f(i)_{1}=\text { the second differential map in the Koszul complex } \mathbb{K}\left(\mathbf{y}_{i 1}\right) \text {. }
\end{aligned}
$$

Let $s=\left(\begin{array}{c}n+2 \\ 2\end{array}\right)$. It follows from part (1) of Theorem 2.2 that every row of $f(i)$ has exactly $(n+1)$ nonzero and $(s-n-1)$ zero entries. Let $S_{k}$ be the $(n+2) \times(n+1)$ submatrix of $f(i)$ formed by $(n+1)$ columns which entries of the $k$ th row of $f(i)$ are nonzero. Let $T_{k}$ be the $(n+2) \times(n+1)$ 
submatrix of $f(i)$ formed by $(s-n-1)$ columns which entries of the $k$ th row of $f(i)$ are zero. We have shown that the conditions of Proposition 4.3 [6] are satisfied. It follows from Theorem 2.2 that the conditions of Theorem $4.4[6]$ are satisfied.

Now we are ready to give a matrix defined by complete matrices $f(i)$ of grade $n+2$.

DeFinition 2.5. Let $R$ be a commutative ring with identity. Let $n$ be an integer with $n \geq 2$ and $x_{i 1}, x_{i 2}, \ldots, x_{i n+2}$ a regular sequence on $R$ for each $i(1 \leq i \leq n)$. Let $f(i)$ be an $(n+2) \times\left(\begin{array}{c}n+2 \\ 2\end{array}\right)$ complete matrix of grade $n+2$ defined in (2.3). We define $f$ to be an $\left(\begin{array}{c}n+2 \\ 2\end{array}\right) \times n(n+2)$ matrix given by

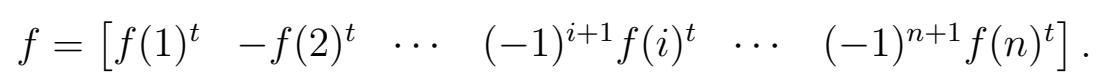

We call $f$ the matrix defined by complete matrices $f(i)$ of grade $n+2$. The following example illustrates Definition 2.5.

EXAMPLE 2.6. Let $x_{i 1}, x_{i 2}, \ldots, x_{i 4}$ be a regular sequence on a commutative ring with identity for each $i$. Then, as shown in Example 2.3, $f(i)^{t}$ has the form

$$
f(i)^{t}=\left[\begin{array}{cccc}
x_{i 2} & x_{i 1} & 0 & 0 \\
-x_{i 3} & 0 & x_{i 1} & 0 \\
x_{i 4} & 0 & 0 & x_{i 1} \\
0 & -x_{i 3} & -x_{i 2} & 0 \\
0 & x_{i 4} & 0 & -x_{i 2} \\
0 & 0 & x_{i 4} & x_{i 3}
\end{array}\right]
$$

We have proved that $f(1)$ and $f(2)$ are complete matrices of grade 4 . The matrix $f$ given by $f=\left[\begin{array}{ll}f(1)^{t}-f(2)^{t}\end{array}\right]$, that is,

$$
f=\left[\begin{array}{cccccccc}
x_{12} & x_{11} & 0 & 0 & -x_{22} & -x_{21} & 0 & 0 \\
-x_{13} & 0 & x_{11} & 0 & x_{23} & 0 & -x_{21} & 0 \\
x_{14} & 0 & 0 & x_{11} & -x_{24} & 0 & 0 & -x_{21} \\
0 & -x_{13} & -x_{12} & 0 & 0 & x_{23} & x_{22} & 0 \\
0 & x_{14} & 0 & -x_{12} & 0 & -x_{24} & 0 & x_{22} \\
0 & 0 & x_{14} & x_{13} & 0 & 0 & -x_{24} & -x_{23}
\end{array}\right]
$$

is a $6 \times 8$ matrix defined by complete matrices $f(1)$ and $f(2)$ of grade 4 .

The following proposition is a consequence of Theorem 4.4 [6].

Proposition 2.7. With notations as above, if $f$ is a matrix defined by complete matrices $f(i)$ of grade $n+2$, then $f$ has exactly $n(n+2)$ 
$(n+1) \times(n+1)$ diagonal submatrices $S_{i j}$ of which the determinant is the $(n+1)$ st power of $x_{i j}$.

Proposition 2.7 enables us to define an ideal associated with the matrix $f$ defined by complete matrices $f(i)$ of grade $n+2$, called the determinantal ideal of an $n \times(n+2)$ matrix $D(f)$.

DeFinition 2.8. Let $R$ be a commutative ring with identity. Let $f$ be a matrix defined by complete matrices $f(i)$ of grade $n+2$ in Definition 2.5 and $x_{i j}$ the $(n+1)$ st root of the determinant of the $(n+1) \times(n+1)$ diagonal submatrix $S_{i j}$ of $f$ mentioned in Proposition 2.7. Let $D(f)=$ $\left(x_{i j}\right)$ be an $n \times(n+2)$ matrix. Let $X_{i j}$ be the element of $R$ defined by

$$
X_{i j}= \begin{cases}\operatorname{det} A_{i j} & \text { if } i<j \\ 0 & \text { if } i=j \\ -\operatorname{det} A_{j i} & \text { if } i>j\end{cases}
$$

, where $A_{i j}$ is the submatrix of $D(f)$ obtained by deleting two columns $i$ and $j$ of $D(f)$. Define $\mathcal{D}_{n+1}(f)$ to be an ideal generated by elements $X_{i j}$, that is,

$$
\mathcal{D}_{n+1}(f)=\left(X_{12}, X_{13}, \ldots, X_{n+1 n+2}\right) .
$$

Now we construct the minimal free resolution $\mathbb{F}$ of $R / \mathcal{D}_{n+1}(f)$ such that the second differential map of $\mathbb{F}$ is an $\left(\begin{array}{c}n+2 \\ 2\end{array}\right) \times n(n+2)$ matrix $f$ defined by complete matrices $f(i)$ of grade $n+2$.

Let $s=\left(\begin{array}{c}n+2 \\ 2\end{array}\right)$. Let $f_{1}$ be a map from $R^{s}$ to $R$ defined by

$$
f_{1}=\left[\begin{array}{llll}
X_{12} & X_{13} & \cdots & X_{n+1 n+2}
\end{array}\right]: R^{s} \rightarrow R,
$$

and $f_{2}$ a map from $R^{n(n+2)}$ to $R^{s}$ defined by

$$
f_{2}=f: R^{n(n+2)} \rightarrow R^{s} .
$$

For $n \geq 2$, let

$$
s(n)=n(n+2)-s+1=\left(\begin{array}{c}
n+1 \\
2
\end{array}\right) .
$$

Finally we construct a map $f_{3}$ from $R^{s(n)}$ to $R^{n(n+2)}$ such that

$$
\mathbb{F}: 0 \longrightarrow R^{s(n)} \stackrel{f_{3}}{\longrightarrow} R^{n(n+2)} \stackrel{f_{2}}{\longrightarrow} R^{s} \stackrel{f_{1}}{\longrightarrow} R
$$

is a minimal free resolution of $R / \mathcal{D}_{n+1}(f)$. Since $\mathbf{x}_{i}=x_{i 1}, x_{i 2}, \ldots, x_{i n+2}$ is a regular sequence,

$$
\mathcal{K}_{n+1}(f(i))=\left(x_{i 1},-x_{i 2}, \ldots,(-1)^{k+1} x_{i k}, \ldots,(-1)^{n+3} x_{i n+2}\right)
$$


is a complete intersection of grade $n+2$ for each $i$. Hence by Theorem 4.10 [6], we have the Koszul complex $\mathbb{K}\left(\tilde{\mathbf{x}}_{i}\right)$ defined by the regular sequence $\tilde{\mathbf{x}}_{i}=x_{i 1},-x_{i 2}, \ldots,(-1)^{k+1} x_{i k}, \ldots,(-1)^{n+3} x_{i n+2}$ such that the second differential map of $\mathbb{K}\left(\tilde{\mathbf{x}}_{i}\right)$ is $f(i)$. For each $i$ we define $\tilde{f}(i)$ to be an $(n+2) \times 1$ matrix given by

$$
\tilde{f}(i)=\left[\begin{array}{llllll}
x_{i 1} & -x_{i 2} & \cdots & (-1)^{k+1} x_{i k} & \cdots & (-1)^{n+3} x_{i n+2}
\end{array}\right]^{t} .
$$

We note that

$$
\left(\begin{array}{c}
n+1 \\
2
\end{array}\right)=\left(\begin{array}{l}
n \\
1
\end{array}\right)+\left(\begin{array}{l}
n \\
2
\end{array}\right)
$$

Let $h_{1}(i)$ be an $n(n+2) \times 1$ matrix defined as follows: we first divide $h_{1}(i)$ by $n(n+2) \times 1$ submatrices of it. The $i$ th $(n+2) \times 1$ submatrix of it is $\tilde{f}(i)$ and other $(n+2) \times 1$ submatrices are zero matrices. Define $h_{1}$ to be an $n(n+2) \times n$ matrix given by

$$
h_{1}=\left[\begin{array}{llllll}
h_{1}(1) & -h_{1}(2) & \cdots & (-1)^{i+1} h_{1}(i) & \cdots & (-1)^{n+1} h_{1}(n)
\end{array}\right] .
$$

Similarly, we define $h_{2}$ to be an $n(n+2) \times\left(\begin{array}{l}n \\ 2\end{array}\right)$ matrix given as follows: Let $h_{2}(k)$ be the $k$ th column of $h_{2}$. We divide $h_{2}(k)$ by $n(n+2) \times 1$ submatrices of it. Let $P(n+2)=\{(i, j) \mid 1 \leq i<j \leq n+2\}$ be the set of pairs of integers. We set the lexicographic order on $P$, that is,

$$
(1,2)<(1,3)<\cdots<(1, n+2)<(2,3)<(2,4)<\cdots<(n+1, n+2) .
$$

Let $\left(k_{1}, k_{2}\right)$ be the $k$ th element in $P(n+2)$. Define the $k_{1}$ th $(n+2) \times 1$ submatrix of $h_{2}(k)$ to be a $(-1)^{k_{2}+1} \tilde{f}\left(k_{2}\right)$, the $k_{2}$ th submatrix of it to be a $(-1)^{k_{1}} \tilde{f}\left(k_{1}\right)$, and other $(n+2) \times 1$ submatrices to be zero. Hence $h_{2}$ is of the form

$$
h_{2}=\left[\begin{array}{llllll}
h_{2}(1) & h_{2}(2) & \cdots & h_{2}(i) & \cdots & h_{2}(n)
\end{array}\right] .
$$

Finally, using (2.6) and (2.7), we define a map $f_{3}$ from $R^{s(n)}$ to $R^{n(n+2)}$ given by

$$
f_{3}=\left[\begin{array}{ll}
h_{1} & h_{2}
\end{array}\right]: R^{s(n)} \rightarrow R^{n(n+2)} .
$$

We show that the sequence $\mathbb{F}$ of free $R$-modules and $R$-maps defined in (2.5) is a complex.

Lemma 2.9. With reference to Definition 2.8 , for $k=1,2, \ldots, n+2$, we have

$$
\sum_{j=1}^{n+2}(-1)^{j+1} x_{i j} X_{j k}=0 \text { for each } i(1 \leq i \leq n)
$$


Proof. Let $f$ be an $s \times n(n+2)$ matrix defined by complete matrices $f(i)$ and $X=D(f)=\left(x_{i j}\right)$ an $n \times(n+2)$ matrix defined in Definition 2.8 , where $s=\left(\begin{array}{c}n+2 \\ 2\end{array}\right)$. Let $X^{(l)}$ be an $n \times(n+1)$ submatrix of $D(f)$ obtained by deleting the $l$ th column of $D(f)$. Define $X(i)^{(k)}$ to be an $(n+1) \times(n+1)$ matrix given by

$$
X(i)^{(k)}=\left[\begin{array}{c}
\mathbf{r}_{i}\left(X^{(k)}\right) \\
X^{(k)}
\end{array}\right]
$$

, where $\mathbf{r}_{i}\left(X^{(k)}\right)$ is the $i$ th row of $X^{(k)}$.

Since $\operatorname{det} X(i)^{(k)}=\sum_{j=1}^{n+2}(-1)^{j+1} x_{i j} X_{j k}$ and $\operatorname{det} X(i)^{(k)}=0$ for each $i$, the result holds.

The following lemma says that $\mathbb{F}$ defined in (2.5) becomes a free complex.

LEMMA 2.10. With notations as above,

(1) $f(i) \tilde{f}(i)=0$ for each $i$.

(2) $f_{i} f_{i+1}=0$ for $i=1,2$.

Proof. (1) Clear.

(2) For $i=1$, it is immediate from Lemma 2.9 and the definitions of $f_{1}$ and $f_{2}$. For $i=2$, it is immediate from part (1) and the constructions of $f_{2}$ and $f_{3}$.

To complete our main result we need two lemmas.

LEMma 2.11. With notations as above, $I_{s-1}(f)$ contains some powers of $X_{i j}$.

Proof. We note that $f_{2}=f$. Let $v=f$. For each $k$ with $1 \leq k \leq s$, we let $v_{k}$ be the submatrix of $v$ obtained by deleting the $k$ th row of it. Let $P(n+2)$ be a set of pairs of integers defined as above and $\left(k_{1}, k_{2}\right)$ the $k$ th element in $P(n+2)$. We show that $I_{s-1}\left(v_{k}\right)$ contains $X_{k_{1} k_{2}}{ }^{n+1}$. It is sufficient to show this for the case $k=1$. The proof for other cases is similar. Let $n=2$. Then $s-1=\left(\begin{array}{l}4 \\ 2\end{array}\right)-1=5$, and $v$ has the following form

$$
v=\left[\begin{array}{cccccccc}
x_{12} & x_{11} & 0 & 0 & -x_{22} & -x_{21} & 0 & 0 \\
-x_{13} & 0 & x_{11} & 0 & x_{23} & 0 & -x_{21} & 0 \\
x_{14} & 0 & 0 & x_{11} & -x_{24} & 0 & 0 & -x_{21} \\
0 & -x_{13} & -x_{12} & 0 & 0 & x_{23} & x_{22} & 0 \\
0 & x_{14} & 0 & -x_{12} & 0 & -x_{24} & 0 & x_{22} \\
0 & 0 & x_{14} & x_{13} & 0 & 0 & -x_{24} & -x_{23}
\end{array}\right] .
$$


We note that $X_{12}=x_{13} x_{24}-x_{14} x_{23}$. Let $v_{1}\left(i_{1}, i_{2}, \ldots, i_{5}\right)$ be the $5 \times 5$ submatrix of $v_{1}$ formed by five columns $i_{1}, i_{2}, \ldots, i_{5}$ of $v_{1}$. Then we have

$$
\operatorname{det} v_{1}(1,2,3,5,6)=x_{14} X_{12}{ }^{2}, \quad \text { and } \operatorname{det} v_{1}(1,2,4,5,6)=x_{13} X_{12}{ }^{2} \text {. }
$$

Therefore

$$
-x_{23} \operatorname{det} v_{1}(1,2,3,5,6)+x_{24} \operatorname{det} v_{1}(1,2,4,5,6)=X_{12}{ }^{3} \in I_{5}(v) \text {. }
$$

Now we consider the case $n>2$. We have three cases to consider: $n=$ $3, n=4$ and $n>4$.

(a) $n=3$. Then $s-1=9$. We observe from Theorem 2.2 that for each $i$, every column and row of $f(i)^{t}$ contain exactly $n+1$ nonzero and two nonzero entries, respectively. The definition of $v$ says that every column and row of $v$ has exactly $n+1$ nonzero and $2 n$ nonzero entries, respectively. Hence it follows from part (3) of Theorem 2.2 that $v_{1}$ has exactly $2 n$ columns having $n$ nonzero entries and exactly $n^{2}$ columns having $n+1$ nonzero entries. Let $B_{1}$ be the submatrix of $v_{1}$ formed by $2 n$ columns of $v_{1}$ having exactly $n$ nonzero entries. Then $B_{1}$ is an $(s-1) \times 2 n$ matrix. For each $i$, let $l_{1}(i)$ be the sequence of the $x_{i}$ 's defined in $(2.4)$ and $l_{11}(i)$ the submatrix of $l_{1}(i)$ obtained by deleting the first column of it. Then $B_{1}$ has the following form:

$$
\begin{aligned}
B_{1} & =\left[\begin{array}{c}
\overline{B_{1}} \\
\mathbf{0}
\end{array}\right], \text { where } \overline{B_{1}}=\left[\begin{array}{llll}
\overline{B_{1}(1)} & \overline{B_{1}(2)} & \ldots & \overline{B_{1}(n)}
\end{array}\right], \\
\overline{B_{1}(i)} & =(-1)^{i+1}\left[\begin{array}{cc}
l_{11}(i)^{t} & \mathbf{0} \\
\mathbf{0} & l_{11}(i)^{t}
\end{array}\right] .
\end{aligned}
$$

It is easy to show that $\operatorname{det}\left(\overline{B_{1}}\right)=X_{12}{ }^{2}$. Let $f(i)_{1}^{t}$ be the submatrix of $f(i)^{t}$ obtained by deleting the first row of it. It follows from Theorem 2.2 that $f(i)_{1}^{t}$ has three columns containing $n+1$ nonzero entries for each $i$ such that by interchanging these three columns and then multiplying the second column by -1 , the three rows of them form a $3 \times 3$ alternating matrix. We denote them by $(-1)^{i+1} f(1)_{i 1}{ }^{t},(-1)^{i+1} f(2)_{i 1}{ }^{t},(-1)^{i+1} f(3)_{i 1}{ }^{t}$, respectively. Let $p, q$, and $r$ be integers with $1 \leq p, q, r \leq 3$ such that only two of them are equal. Let $i, j$, and $k$ be integers with $1 \leq i, j, k \leq 3$ such that either all of them are distinct or only two of them are equal. Let $B_{2}\left(i_{p}, j_{q}, k_{r}\right)$ be the submatrix of $v_{1}$ formed by three columns $(-1)^{i+1} f(p)_{i 1}{ }^{t},(-1)^{j+1} f(q)_{j 1}{ }^{t}$ and $(-1)^{k+1} f(r)_{k 1}{ }^{t}$ of $v_{1}$. Now we define $B\left(i_{p}, j_{q}, k_{r}\right)$ to be an $(s-1) \times(s-1)$ submatrix of $v_{1}$ given by

$$
B\left(i_{p}, j_{q}, k_{r}\right)=\left[\begin{array}{ll}
B_{1} & B_{2}\left(i_{p}, j_{q}, k_{r}\right)
\end{array}\right] .
$$


We show that if $I_{s-1}(B)$ is an ideal generated by the determinants of the $(s-1) \times(s-1)$ submatrices $B\left(i_{p}, j_{q}, k_{r}\right)$ of $v_{1}$, then $I_{s-1}(B)$ contains $X_{12}{ }^{4}$. We define $X\left(s_{1}, s_{2}, \ldots, s_{a} \mid t_{1}, t_{2}, \ldots, t_{b}\right)$ to be the submatrix of $X$ formed by rows $s_{1}, s_{2}, \ldots, s_{a}$ and columns $t_{1}, t_{2}, \ldots, t_{b}$ of $X$. Now we set

$$
\begin{aligned}
& D_{13}=\operatorname{det} X(2,3 \mid 4,5), D_{23}=\operatorname{det} X(1,3 \mid 4,5), D_{33}=\operatorname{det} X(1,2 \mid 4,5) \\
& D_{14}=\operatorname{det} X(2,3 \mid 3,5), D_{24}=\operatorname{det} X(1,3 \mid 3,5), D_{34}=\operatorname{det} X(1,2 \mid 3,5) .
\end{aligned}
$$

Then we have

$$
X_{12}=x_{13} D_{13}-x_{23} D_{23}+x_{33} D_{33}=-x_{14} D_{14}+x_{24} D_{24}-x_{34} D_{34} .
$$

Let $\tilde{B}_{2}\left(i_{p}, j_{q}, k_{r}\right)$ be the submatrix of $B_{2}\left(i_{p}, j_{q}, k_{r}\right)$ formed by the last three rows of $B_{2}\left(i_{p}, j_{q}, k_{r}\right)$. Then it follows from (2.8) and the determinant of the block matrix that

$$
\operatorname{det} B\left(i_{p}, j_{q}, k_{r}\right)=X_{12}^{2} \operatorname{det} \tilde{B}_{2}\left(i_{p}, j_{q}, k_{r}\right) .
$$

The following simple computation shows that $X_{12}^{2}$ is a linear combination of elements $\operatorname{det} \tilde{B}_{2}\left(i_{p}, j_{q}, k_{r}\right)$ :

$$
\begin{aligned}
- & x_{13} x_{14} D_{13} D_{14}-x_{23} x_{24} D_{23} D_{24}-x_{33} x_{34} D_{33} D_{34} \\
= & -\operatorname{det} B\left(1_{3}, 2_{1}, 3_{1}\right) \operatorname{det} B\left(1_{3}, 2_{2}, 3_{2}\right)-\operatorname{det} B\left(1_{1}, 2_{3}, 3_{1}\right) \operatorname{det} B\left(1_{2}, 2_{3}, 3_{2}\right) \\
& -\operatorname{det} B\left(1_{1}, 2_{1}, 1_{3}\right) \operatorname{det} B\left(1_{2}, 2_{2}, 3_{3}\right),
\end{aligned}
$$

and

$$
\begin{aligned}
x_{13} x_{24} D_{13} D_{24} & =-x_{13} x_{24} x_{33} \operatorname{det} B\left(1_{2}, 2_{1}, 3_{1}\right)+x_{13}^{2} x_{35} \operatorname{det} B\left(2_{1}, 2_{3}, 3_{1}\right), \\
-x_{13} x_{34} D_{13} D_{34} & =-x_{13} x_{34}^{2} \operatorname{det} B\left(1_{2}, 2_{1}, 2_{2}\right)+x_{13} x_{24} x_{34} \operatorname{det} B\left(1_{2}, 2_{2}, 3_{1}\right), \\
x_{14} x_{23} D_{14} D_{23} & =x_{14} x_{23} x_{33} \operatorname{det} B\left(1_{1}, 2_{2}, 3_{1}\right)-x_{14} x_{23}^{2} \operatorname{det} B\left(1_{1}, 3_{1}, 3_{2}\right), \\
x_{23} x_{34} D_{14} D_{23} & =x_{15} x_{34}^{2} \operatorname{det} B\left(1_{2}, 2_{2}, 2_{3}\right)-x_{15} x_{2,3} x_{35} \operatorname{det} B\left(1_{2}, 2_{2}, 3_{1}\right), \\
-x_{14} x_{33} D_{14} D_{33} & =-x_{14} x_{33}^{2} \operatorname{det} B\left(1_{1}, 2_{1}, 2_{2}\right)-x_{13} x_{24} x_{35} \operatorname{det} B\left(1_{2}, 2_{2}, 3_{1}\right), \\
x_{24} x_{33} D_{24} D_{33} & =-x_{24} x_{33}^{2} \operatorname{det} B\left(1_{1}, 1_{2}, 2_{1}\right)+x_{13} x_{24} x_{33} \operatorname{det} B\left(1_{1}, 2_{1}, 3_{2}\right) .
\end{aligned}
$$

Hence it follows from (2.9) and (2.10) that $X_{12}{ }^{4}$ is contained in the ideal $I_{s-1}(B)$.

(b) $n=4$. Then $s-1=14$. As shown in the case of $n=3$, Theorem 2.2 states that every column and row of $v$ has exactly $n+1$ nonzero and $2 n$ nonzero entries, respectively. Let $B_{1}, \bar{B}_{1}$ and $l_{11}(i)$ be the matrices defined as in the case of $n=3$. Direct computation shows that $\operatorname{det}\left(\bar{B}_{1}\right)=X_{12}{ }^{2}$. Since $x_{i 3},-x_{i 4}, x_{i 5},-x_{i 6}$ is a regular subsequence of the regular sequence $x_{i 1},-x_{i 2}, x_{i 3},-x_{i 4}, x_{i 5},-x_{i 6}$ for each $i$, by Theorem 3.5 [11], there exists a $4 \times 6$ complete submatrix $f(i)$ of a complete matrix 
$f(i)$ of grade 6 such that $\mathcal{K}_{3}(\check{f}(i))=x_{i 3},-x_{i 4}, x_{i 5},-x_{i 6}$ is a complete intersection of grade 4. $f(i)$ has the following form

$$
\check{f(i)^{t}}=\left[\begin{array}{cccc}
-x_{i 4} & -x_{i 3} & 0 & 0 \\
x_{i 5} & 0 & -x_{i 3} & 0 \\
-x_{i 6} & 0 & 0 & -x_{i 3} \\
0 & x_{i 5} & x_{i 4} & 0 \\
0 & -x_{i 6} & 0 & x_{i 4} \\
0 & 0 & -x_{i 6} & -x_{i 5}
\end{array}\right]
$$

Hence $v_{1}$ contains exactly four columns such that the first three entries of the last six entries of them are nonzero, and the second three entries of them are zero ( For example, see the first column of $\left.f(i)^{t}\right)$. Let $C_{2}\left(l_{1}, l_{2}, l_{3}\right)$ be the $(s-1) \times 3$ submatrix of $v_{1}$ formed by three columns $l_{1}, l_{2}, l_{3}$ of the above four columns in this order. Let $B_{2}\left(i_{p}, j_{q}, k_{r}\right)$ be the $(s-1) \times 3$ submatrix of $v_{1}$ defined as in the case of $n=3$. Now we define $B\left(l_{1}, l_{2}, l_{3}, i_{p}, j_{q}, k_{r}\right)$ to be an $(s-1) \times(s-1)$ submatrix of $v_{1}$ given by

$$
B\left(l_{1}, l_{2}, l_{3}, i_{p}, j_{q}, k_{r}\right)=\left[\begin{array}{lll}
B_{1} & C_{2}\left(l_{1}, l_{2}, l_{3}\right) & B_{2}\left(i_{p}, j_{q}, k_{r}\right)
\end{array}\right] .
$$

Let $\tilde{B}_{2}\left(i_{p}, j_{q}, k_{r}\right)$ be the submatrix of $v_{1}$ defined as in the case of $n=3$. Let $\tilde{C}_{2}\left(l_{1}, l_{2}, l_{3}\right)$ be the submatrix of $v_{1}$ consisting of the second three nonzero entries of the last six entries of $\mathbf{c}\left(v_{1}\right)$ described as above. Then

$$
\operatorname{det} B\left(l_{1}, l_{2}, l_{3}, i_{p}, j_{q}, k_{r}\right)=X_{12}^{2} \operatorname{det} \tilde{C}_{2}\left(l_{1}, l_{2}, l_{3}\right) \operatorname{det} \tilde{B}_{2}\left(i_{p}, j_{q}, k_{r}\right) .
$$

Similarly to the case of $n=3$, if $I_{s-1}(B)$ is the ideal generated by the determinants of the submatrices $B\left(l_{1}, l_{2}, l_{3}, i_{p}, j_{q}, k_{r}\right)$, then it contains $X_{12}{ }^{5}$.

(c) $n>4$. Similarly to the case of $n=4$, we can see that if $I_{s-1}(B)$ is the ideal generated by the determinants of the submatrices of $v_{1}$ defined as in the case of $n=4$, then $I_{s-1}(B)$ contains $X_{12}^{n+1}$.

LEMMA 2.12. With notations as above, $I_{s(n)}\left(f_{3}\right)$ contains some powers of $X_{i j}$ for every $i<j$.

Proof. Let $P(n+2)$ be the set of pairs of integers defined as above and $\left(k_{1}, k_{2}\right)$ the $k$ th element in $P(n+2)$. Let $w=f_{3}$ and $w_{k}$ an $n^{2} \times s(n)$ submatrix of $w$ obtained by deleting rows $k_{1}, k_{2},(n+2)+k_{1},(n+2)+$ $k_{2}, \ldots,(n-1)(n+2)+k_{1},(n-1)(n+2)+k_{2}$ of $w$. Similarly to Lemma 2.11 , we can show that $I_{s(n)}\left(w_{k}\right)$ contains $X_{k_{1} k_{2}}{ }^{n}$ for each $k$. In the proof of Lemma 2.11 we used the column expansion of the determinant $X_{k_{1} k_{2}}$ but in this lemma we perform the row expansion of its determinant. 
The following theorem is our main result.

THEOREM 2.13. Let $R$ be a noetherian local ring with maximal ideal $\mathfrak{m}$. With the notation as above $\mathbb{F}$ is the minimal free resolution of $R / I_{n}(D(f))$ such that the second differential map of $\mathbb{F}$ is a matrix $f$ defined by the complete matrices $f(i)$ of grade $n+2$.

Proof. In Lemma 2.10 we proved that $\mathbb{F}$ is a complex. In Lemmas 2.11 and 2.12 we also showed that the rank and depth conditions in the Buchsbaum and Eisenbud's acyclicity criterion [3] are satisfied. Since $x_{i 1}, x_{i 2}, \ldots, x_{i n+2}$ is a regular sequence for each $i$, every $x_{i k}$ is contained in $\mathfrak{m}$ for every $k$. Hence $\mathbb{F}$ is minimal. It is obvious that the second differential map of $\mathbb{F}$ is $f$.

We finish this section with the following example illustrating Theorem 2.13 .

EXAMPLE 2.14. Let $R=\mathbb{Q}[[x, y, z, u, w]]$ be the formal power series ring over the field $\mathbb{Q}$ of rational numbers with indeterminates $x, y, z, u, w$. We note that $x, y, z, u, w$ is a regular sequence. Let $f(1), f(2)$ and $f(3)$ be $5 \times 10$ matrices given by

$$
\begin{gathered}
f(1)=\left[\begin{array}{ccccc}
-y & -x & 0 & 0 & 0 \\
z & 0 & -x & 0 & 0 \\
-u & 0 & 0 & -x & 0 \\
w & 0 & 0 & 0 & -x \\
0 & z & y & 0 & 0 \\
0 & -u & 0 & y & 0 \\
0 & w & 0 & 0 & y \\
0 & 0 & -u & -z & 0 \\
0 & 0 & w & 0 & -z \\
0 & 0 & 0 & w & u
\end{array}\right], f(2)=\left[\begin{array}{cccccc}
u & z & 0 & 0 & 0 \\
-x & 0 & z & 0 & 0 \\
w & 0 & 0 & z & 0 \\
-y & 0 & 0 & 0 & z \\
0 & -x & -u & 0 & 0 \\
0 & w & 0 & -u & 0 \\
0 & -y & 0 & 0 & -u \\
0 & 0 & w & x & 0 \\
0 & 0 & -y & 0 & x \\
0 & 0 & 0 & -y & -w
\end{array}\right]^{t}, \\
f(3)=\left[\begin{array}{ccccc}
-x & -w & 0 & 0 & 0 \\
y & 0 & -w & 0 & 0 \\
-z & 0 & 0 & -w & 0 \\
u & 0 & 0 & 0 & -w \\
0 & y & x & 0 & 0 \\
0 & -z & 0 & x & 0 \\
0 & u & 0 & 0 & x \\
0 & 0 & -z & -y & 0 \\
0 & 0 & u & 0 & -y \\
0 & 0 & 0 & u & z
\end{array}\right] . \\
\end{gathered}
$$


Then they are complete matrices of grade 5 . Moreover $\tilde{f}(1), \tilde{f}(2)$, and $\tilde{f}(3)$ are given by

$$
\begin{aligned}
\tilde{f}(1) & =\left[\begin{array}{lllll}
x & -y & z & -u & w
\end{array}\right]^{t}, \quad \tilde{f}(2)=\left[\begin{array}{lllll}
z & -u & x & -w & y
\end{array}\right]^{t}, \\
\tilde{f}(3) & =\left[\begin{array}{lllll}
w & -x & y & -z & u
\end{array}\right]^{t} .
\end{aligned}
$$

Let $f$ be a $10 \times 15$ matrix defined by complete matrices $f(1), f(2)$, and $f(3)$, that is

$$
f=\left[\begin{array}{lll}
f(1)^{t} & -f(2)^{t} & f(3)^{t}
\end{array}\right] .
$$

Then $x, y, z, u$, and $w$ are the fourth roots of the determinants of the $4 \times 4$ diagonal submatrices of $f(i)$ for each $i$. Let $X=D(f)$ be a $3 \times 5$ matrix defined in Definition 2.8, that is,

$$
D(f)=\left[\begin{array}{ccccc}
x & -y & z & -u & w \\
z & -u & x & -w & y \\
w & -x & y & -z & u
\end{array}\right]
$$

Let $X_{i j}$ be the determinant of the submatrix of $D(f)$ obtained by deleting two columns $i, j$ of it. Thus $\mathcal{D}_{4}(f)$ is generated by the elements $X_{i j}$. The minimal free resolution $\mathbb{F}$ of $R / \mathcal{D}_{4}(f)$ is

$$
\mathbb{F}: 0 \longrightarrow R^{6} \stackrel{f_{3}}{\longrightarrow} R^{15} \stackrel{f_{2}}{\longrightarrow} R^{10} \stackrel{f_{1}}{\longrightarrow} R,
$$

where

$$
\begin{aligned}
& f_{1}=\left[\begin{array}{llllllllll}
X_{12} & X_{13} & X_{14} & X_{15} & X_{23} & X_{24} & X_{25} & X_{34} & X_{35} & X_{45}
\end{array}\right], \\
& f_{2}=\left[\begin{array}{lll}
f(1)^{t} & -f(2)^{t} & f(3)^{t}
\end{array}\right] \\
& f_{3}=\left[\begin{array}{cccccc}
-\tilde{f}(1) & \mathbf{0} & \mathbf{0} & \mathbf{0} & \tilde{f}(3) & -\tilde{f}(2) \\
\mathbf{0} & \tilde{f}(2) & \mathbf{0} & \tilde{f}(3) & \mathbf{0} & \tilde{f}(1) \\
\mathbf{0} & \mathbf{0} & -\tilde{f}(3) & -\tilde{f}(2) & -\tilde{f}(1) & \mathbf{0}
\end{array}\right] .
\end{aligned}
$$

Clearly the second differential map of $\mathbb{F}$ is $f$ and $\mathcal{D}_{4}(f)=I_{3}(\tilde{X})$, where

$$
\tilde{X}=\left[\begin{array}{lllll}
x & y & z & u & w \\
z & u & x & w & y \\
w & x & y & z & u
\end{array}\right]
$$

\section{References}

[1] K. Akin, D.A. Buchsbaum, and J.Weyman, Resolutions of determinantal ideals, Adv. Math. 44 (1981), 1-30. 
[2] D.A. Buchsbaum and D. Eisenbud, Remarks on ideals and Resolutions, Symposia Math XI (1973), 193-204, Acadamic Press, London.

[3] D.A. Buchsbaum and D. Eisenbud, What makes the complex exact?, J. Algebra 25 (1973), 259-268.

[4] D.A. Buchsbaum and D.S. Rim, A generalized Koszul complex. II, Proc. Amer. Math. Soc. 16 (1965), 197-225.

[5] D.A. Buchsbaum, A new construction of the Eagon-Northcott complex, Adv. Math. 34 (1979), 58-76.

[6] E.J. Choi, O.J. Kang and H.J. Ko, A structure theorem for complete intersections, Bull. Korean Math. Soc. 46 (2009), no 4, 657-671.

[7] J.A. Eagon and D.G. Northcott, Ideals defined by matrices and a certain complex associated with them, Proc. R. Soc. Lond. Ser. A Math. Phys. Eng. Sci. 269 (1962), 188-204.

[8] M. Hashimoto, Determinantal ideals without minimal free resolutions, Nagoya Math. J. 118 (1990), 203-216.

[9] M. Hashimoto, Resolutions of determinantal ideals :t-Minors of $(t+2) \times n M a-$ trices, J. Algebra 142 (1991), 456-491.

[10] M. Hashimoto and K. Kurano, Resolutions of Determinantal ideals: $n$-minors of $(n+2)$-Square Matrices, Adv. Math. 94 (1992), 1-66.

[11] O.J. Kang and H.J. Ko, The structure theorem for complete intersections of grade 4, Algebra Colloq. 12 (2) (2005), 181-197.

[12] A. Lascoux, Syzygies des varietes determinantales, Adv. Math. 30 (1978), 202 237.

[13] P. Pragacz and J. Weyman, Complexes associated with trace and evaluation: another approach to Lascoux's resolution, Adv. Math. 57 (1985), 163-207.

[14] P. Roberts, A minimal free complex associated to minors of a matrix, Preprint.

Department of Mathematics

School of Natural Sciences

University of Incheon

402-749 Incheon, Korea

E-mail: ohkang@incheon.ac.kr

Department of Mathematics Education

Wonkwang University

570-749 Iksan, Korea

E-mail: joohyung@wku.ac.kr 